\title{
ESTUDO SOBRE PROGRAMAS DE PREPARO DA EQUIPE DE ENFERMAGEM: FASES IMPLEMENTADAS NOS HOSPITAIS CAMPO DE PRÁTICA DA ESCOLA DE ENFERMAGEM DA UNIVERSIDADE DE SÃO PAULO*
}

\author{
Maria Madalena Janudrio Leite**
}

LEITE, M.M.J. Estudo sobre programas de preparo da equipe de enfermagem: fases implementadas nos hospitais campo de prática da Escola de Enfermagem da Universidade de Sáo Paulo. Rev. Esc. Enf. USP, Sào Paulo, 22(2):189-198, ago. 1988.

A autora faz um levantamento das fases e respectivas atividades que compoem os programas de de. senvolvimento do pessoal de enfermagem, realizados pelos hospitais campo de prática da Escola de En. fermagem da Universidade de Sdo Paulo.

UNITERMOS: Educação em serviço. Serviços de enfermagem hospiralar. Treinamento em serviço.

\section{INTRODUÇÃO}

A relação homem-organização tem sido influenciada por fatores de ordem econômica, social e cultural e sua evolução é analisada, historicamente, por CHIAVENATO', DRAKE ${ }^{15}$ e MOTTA ${ }^{29}$.

Segundo esses autores, com a revolução industrial e devido à utilização das máquinas, o processo de produção passou a ser realizado em larga escala, favorecendo, dessa forma, a valorização do planejamento e da racionalização dos métodos de trabalho. Ainda nessa fase, os incentivos salariais passaram a ser considerados essenciais para melhor produtividade do empregado.

Com o desenvolvimento econômico advindo do periodo pós-guerra, na década de 40, surgiram os movimentos sindicais que pleiteavam melhores condiçסes de trabalho, e, a partir desse periodo, foram elaboradas teorias no campo de administração, voltadas para o estudo da motivação e da sua interferência na dinâmica organizacional.

Entre os vários estudiosos dessa fase destacam-se Argyris, Likert, McGregor e Simon. Para eles o empregado pode ser motivado quando a organização permite autonomia na tomada de decisão e favorece o desenvolvimento e a satisfação no trabalho.

Com as teorias $\mathrm{X}$ e $\mathrm{Y}$ postuladas por McGREGOR ${ }^{27}$, a relação homem-organização passou a receber um novo enfoque. Segundo a teoria $X$, o homem poderia ser

- Resumo da Dissertação de Mestrado apresentado à Escola de Enfermagem da USP, 1985.

- Enfermeira. Mestre em Enfermagem. Professor Assistente do Departamento de Orientaçăo Profissional - disciplina Administraçào Aplıcada à Enfermagem. 
motivado por incentivos económicos, sendo considerado preguiçoso e irracional, cabendo à organizaçăo dirigir seus empregados por meio de ameaças, puniçðes e disciplinas rígidas.

Por outro lado, a teoria $\mathrm{Y}$ preconizou que o homem poderia se desenvolver, tendo capacidade de assumir responsabilidades, e sua passividade seria decorrente das experiências vivenciadas em outras organizaçoes. Dessa forma seria de responsabilidade da organização criar oportunidades de orientaçăo e crescimento aos seus empregados, propiciando satisfação no trabalho.

Com a teoria geral dos sistemas, publicada por Berta Lanfky na década de 60 , as organizaçðes passaram a ser consideradas, segundo KATZ ${ }^{20}$ e NASCIMENTO como um conjunto de partes interrelacionadas, que influenciariam e seriam influenciadas pelo meio ambiente, ocorrendo uma relaçào de interdependência entre o homem, a organização e a sociedade.

CHIAVENATO ${ }^{8}$ mostrou que atualmente as organizaçðes têm sido estudadas dentro do enfoque sistêmico como um conjunto de cinco variáveis (tarefa, estrutura, empregados, tecnologia e ambiente) que interagem e são interdependentes.

Para BERGAMINI ${ }^{3}$ e PETERS \& WATERMAN ${ }^{3 !}$, as organizaçōes passaram a valorizar o ambiente de trabalho como um dos fatores que propiciam ao empregado maior satisfação e auto-realização.

Autores como MEJIA \& FULOP ${ }^{2 x}$ e TEJADA DE RIVERO ${ }^{40}$ consideraram que caberia, às organizaçðes de saúde, a promoçảo de oportunidades de ensino e formação adequada para que o seu pessoal desenvolva, com eficiência, atividades produtivas junto à população.

Os Serviços de Enfermagem, nessas organizaçòes, têm demonstrado preocupação crescente em realizar atividades voltadas para o desenvolvimento do pessoal de enfermagem, embora tenha sido observada a não existência de filosofia que norteie o planejamento e execução de tais atividades. Estas, mesmo nåo estando inseridas numa política adequada de recursos humanos, têm sido agrupadas e organizadas em setores ou serviços denominados "treinamento" ou "educaçào em serviço".

Autores como CAMARGO', CASTRO', DEIMAN', DI VICENTI ${ }^{14}$, NŨNEZ \& LUCKES ${ }^{32}$ descreveram a educação em serviço como um órgào que objetiva propiciar experiências educativas no local de trabalho, de forma planejada e continua, oferecendo ao empregado oportunidades de desenvolvimento pessoal e profissional.

COOPER", entretanto, ressaltou que a educação em serviço desenvolveu-se lentamente sendo um dos principais fatores dessa lentidảo a insatisfaçāo da equipe de enfermagem com seus programas, o que evidenciou sua inadequação.

Para vários autores a eficácia dos programas depende do planejamento cuidadoso e detalhado.

CHIAVENATO ${ }^{7}$, COOMBS $^{\prime \prime}$, COOPER \& HORNBACK ${ }^{\prime \prime}$, DAVIES $^{\prime 2}$ e POPIEL ${ }^{36}$ enfatizaram a importância do planejamento dos programas, uma vez que envolve a determinação de objetivos e a escolha de alternativas, de acordo com os recursos humanos e materiais disponiveis. Como consequiência, as decisðes sào otimizadas, tornando os programas mais efetivos, com maiores possibilidades de atender satisfatoriamente à clientela e aos profissionais que desenvolvem o programa.

Ainda segundo esses autores e outros como DI VICENTI' ${ }^{14}$, ESCOBAR ${ }^{16}$, NŨNEZ \& LUCKES $^{32}$, AQUINO', PARO ${ }^{33}$ e SANTOS ${ }^{31}$, o planejamento dos programas de desenvolvimento de pessoal compóe-se de várias fases: "levantamento de necessidades e de diagnóstico", "elaboração, aprovação, execução e avaliação do programa", e "elaboração do relatório". 
Para esses autores a fase de levantamento de necessidades e de diagnóstico é extremamente importante, uma vez que são verificadas as necessidades sentidas e evidenciadas pelos funcionários $\mathrm{e}$ as identificadas no seu desempenho técnico.

DI VICENTI I ${ }^{14}$ definiu a necessidade sentida como a percebida pelo funcionário e a identificada como a observada no seu desempenho. A necessidade evidenciada foi conceituada por KURCGANT ${ }^{21}$ como aquela manifestada ou demonstrada pelo funcionário diante de uma situação que the é proposta.

A fase de elaboração do programa, segundo AQUINO', consiste na definição de objetivos, na escolha do conteúdo programático e das estratégias de ensino. Para esse autor é uma fase difícil de ser realizada, uma vez que deve corresponder às necessidades identificadas na fase anterior.

Quanto à fase de execução do programa consiste para CHIAVEENATO', na realização do programa planejado e na identificação dos pontos críticos e falhas que estão ocorrendo.

DI VICENTI ${ }^{14}$ e POPIEL ${ }^{36}$ mostraram a necessidade da fase de avaliação do programa, uma vez que ela conduz à análise dos resultados do programà executado, possibilitando a efetivação dos futuros programas.

Considerando a importância dos programas de desenvolvimento de pessoal para o crescimento da equipe de enfermagem, decidiu a autora fazer um estudo que verificasse as fases dos programas realizados pela Educação em Serviço dos "hospitais campo de prática" da Escola de Enfermagem da USP e os recursos humanos e materiais disponiveis para a realização desses programas.

\section{METODOLOGIA}

Como universo do estudo foram considerados os 12 hospitais que são campo de prática dos estudantes das disciplinas que integram os Cursos de Graduação em Enfermagem e Obstetricia, de Habilitação em Enfermagem Obstétrica e de Especialização em Enfermagem de Cuidados Intensivos, da Escola de Enfermagem da Universidade de São Paulo (EEUSP).

Foi considerado critério para a seleção desses hospitais o fato de apresentarem, na sua estrutura administrativa, um órgão de Educação em Serviço que contasse com, pelo menos, uma enfermeira atuando especificamente nessa área.

Para coleta de dados foi solicitado à enfermeira responsável pela Educação em Serviço que respondesse questðes contidas num formulário, referentes aos seguintes itens: "identificação dos hospitais", "caracterização do orgão de Educação em Serviço", "caracterizaçāo da enfermeira responsável pela Educação em Serviço" e "fases e atividades dos programas de preparo de pessoal realizados pela Educação em Serviço".

Para a elaboração desse formulário foram estabelecidas as fases e as respectivas atividades do programa de desenvolvimento do pessoal de enfermagem, descritas pela literatura e encontradas na prática.

Essas fases e atividades foram analisadas segundo os critérios "essencialidade" e "conveniência", determinados no estudo.

Como essencialidade compreendeu-se a fase e a atividade que são indispensáveis ao desenvolvimento do programa e, como conveniência, entendeu-se a fase e a atividade que interferem diretamente na eficácia do programa. 
Foram atribuidos valores às fases e às atividades, de acordo com esses critérios, resultando a somatória num valor pré-determinado de 119 pontos, agrupados em 3 intervalos: de 119 a 80 pontos, de 79 a 40 pontos e de 39 a 0 pontos, denominados respectivamente em grupos 1 , II e III.

Após a aplicação do formulário, cada hospital, de acordo com os pontos obtidos, foi classificado em um dos 3 grupos.

Foram adotadas as abreviaçðes HGI, HGIl e HGIII para identificar os hospitais classificados nos grupos 1 , II e III, respectivamente.

$\mathrm{O}$ quadro abaixo mostra os valores atribuídos às fases e respectivas atividades que conpoem os programas de preparo de pessoal.

\section{QUADRO I}

FASE E ATIVIDADES COMPONENTES DOS PROGRAMAS DE EDUCAÇĀO EM SERVIÇO. COM OS RESPECTIVOS VALORES ATRIBUIDOS.

19a Fase: Levantamento de necessidade e de diagnóstico

Valor

Atribuído

- Caracterização da clientela alvo

- Identificação das necessidades sentidas pela clientela do programa

- Identificação das necessidades evidenciadas

- Verificação do desempenho da clientęla

- Análise dos dados coletados

- Priorização das necessidades

- Identificação das atividades que compōem a função ou cargo ocupado pela clien tela alvo

- Identificação dos conhecimentos, habilidades e atitudes necessários para a execução das atividades que compōem a função ou cargo

- Análise dos recursos humanos e materiais

- Análise da disponibilidade de tempo para a determinação da carga horária e do período de realização do programa

- Definição dos objetivos a serem alcançados ao final do programa

- Determinação do conteúdo programático

- Estabelecimento das estratégias de ensino

- Definição do processo de avaliação discente

- Definição do processo de avaliação docente 
- Coordenação e execução das atividades programadas

\section{ANȦLISE E DISCUSSĀO DOS RESULTADOS}

Após a classificação dos hospitais de acordo com os pontos obtidos, segundo as fases e atividades por eles desenvolvidas nos programas de preparo de pessoal, verificou-se que $4(33,3 \%)$ dos $12(100 \%)$ hospitais pesquisados foram classificados no GI, $8(66,7 \%)$ no GII e nenhum no GIII.

Nenhum dos hospitais estudados foi classificado no Grupo III, uma vez que todos desenvolveram as fases "levantamento de necessidades e de diagnóstico", "elaboração do programa", "aprovação do programa" e "execução do programa", consideradas, conforme o critério essencialidade, fundamentais na realizaçào de programas.

HEADRICKS ${ }^{18}$; TURRA ${ }^{43}$ e WOLANIN"4 mostraram que a 1 , fase, "levantamento de necessidades e de diagnostico', facilita a elaboração dos programas, auxi- 
liando na definição dos objetivos, na seleção dos conteúdos e na escolha de estratégias de ensino. É nesta fase que são identificados os interesses, as expectativas e as metas da clientela, consideradas fundamentais na obtençăo de resultados satisfatórios.

Sobre a 3a fase, "elaboração do programa", AQUINO', DAVIES"2, MARCONDES $^{25}$ e PARO ${ }^{35}$ consideraram-na como a estrutura dos programas de preparo de pessoal, uma vez que nela se define "o quê" e "o como" ensinar e qual o comportamento final desejado.

Em relação à 4" fase, "aprovação do programa", COOPER \& HORNBACK", HORN ${ }^{19}$ e MARCONDES ${ }^{25}$ evidenciaram a sua importância, quando mostraram que o sucesso dos programas depende tanto da aceitação por parte da clientela como por parte da chefia, o que favorece a obtenção de bons resultados.

A 5? fase, "execução do programa, foi considerada a de maior grau de essencialidade, tendo sido a ela atribuido maior número de pontos, uma vez que sua ausência significa a não realização do programa. Autores como DAVIES ${ }^{12}, \mathrm{LEWY}^{22} \mathrm{e}$ TURRA ${ }^{42}$ enfatizaram a importância desta fase, relatando que a forma cọmo é conduzido um programa interfere diretamente na eficácia dos resultados.

A 6: e a 7: fases "avaliação do programa executado" e "elaboração do relatório final" foram realizadas com menor frequiência, pelos hospitais pesquisados e a 2: fase, "análise das atividades referentes às funçðes e conhecimentos da clientela alvo", não foi desenvolvida por qualquer dos hospitais do estudo. Esses resultados podem ser explicados pelo fato destas três fases não apresentarem a característica essencialidade e, portanto, quando ausentes, não impedirem a operacionalizaçāo dos programas.

A fase "análise das atividades referentes às funçðes e conhecimentos da clientela alvo" foi considerada de grande importância por CAMACHO ${ }^{4}$, FONTES", SMITH $^{39}$ e TOLEDO ${ }^{41}$, uma vez que é necessária para a definição do conteúdo programático e para a seleção das experiências da aprendizagem. Segundo tais autores, a inexistência dessa fase tem, como conseqüência, o năo atendimento das necessidades da clientela, resultando em frustração dos funcionários para os quais foi direcionado o programa.

Outro aspecto que evidenciou a importância da realização dessa fase pela Educação em Serviço é a heterogeneidade na formação da equipe de enfermagem cujos elementos desenvolvem atividades de natureza e abrangências diversas; portanto, a enfermeira da Educação em Serviço, ao analisar as atividades realizadas pelo pessoal de enfermagem, tem maiores condiçð̌es de adequar o conteúdo do programa aos conhecimentos e habilidades exigidas para o desenvolvimento dessas atividades.

A fase "avaliação do programa executado" foi comentada por BANDEIRA?2, CHIAVENATO ${ }^{7}$, COOPER \& HORNBACK ${ }^{\prime \prime}$, DAVIES ${ }^{12}$, DI VICENTI ${ }^{14}$, HORN $^{19}$, LEWY ${ }^{22}$, LUCKESI ${ }^{24}$, MARRINER $^{26}$, POPIEL ${ }^{36} \mathrm{e}^{\text {TURRA }}{ }^{43}$. Para tais autores, essa fase fornece informaçð̄es fundamentais para planejamento dos futuros programas, possibilitando o alcance dos objetivos propostos com economia de tempo e de recursos.

A fase "elaboração do relatório" é necessária para subsidiar a chefia nas decisões relativas à administração de pessoaol, ao planejamento orçamentário e à previsåo de recursos humanos e materiais, uma vez que nessa fase são descritos os resultados dos programas implementados. 
Evidenciou-se, ainda, neste estudo que os $\mathrm{HGI}$, em relação aos HGII, desenvolveram, com maior frequêencia, atividades que compõem as fases dos programas de preparo de pessoal, obtendo portanto maior número de pontos.

Quanto à caracterização dos hospitais do estudo, verificou-se que os $\mathrm{HGl}$ e HGII não diferiram em relaçảo ao tipo de entidade mantenedora, mas apresentaram resultados diversos quanto ao tipo de assistência prestada e à capacidade operacional.

Observou-se que a maioria dos $\mathrm{HGl}$ eram públicos, gerais e de tamanho extra, enquanto grande parte dos HGll eram públicos, especializados e de grande porte.

Autores cono KAST" e LITTERER" consideraram que as organizaçðes maiores caracterizam-se pelo alto grau de especialização e maior divisão de trabalho e que existe relação positiva entre o tamanho da instituição e do desempenho técnico dos funcionários.

Sobre a estrutura administrativa dos órgãos de Educação em Serviço, constatou-se que em todos os $\mathrm{HGl}$ e em grande parte dos HGII são eles subordinados às chefias de enfermagem. E fundamental, para a efetividade dos programas, que a Educação em Serviço reporte-se administrativa e tecnicamente ao Serviço de Enfermagem, tendo, inclusive, a coordenação própria e autonomia na tomada de decisðes.

Quanto aos recursos humanos disponiveis para a Educação em Serviço, evidenciou-se neste estudo, que os $\mathrm{HGI}$ apresentaram, em relação aos HGIl, maior número de enfermeiras atuando especificamente na Educaçăo em Serviço. Verificou-se que os $\mathrm{HGI}(100 \%)$ contavam com duas a quatro enfermeiras, enquanto nos $8 \mathrm{HGII}$ $(100 \%)$ só havia uma enfermeira para atividades especificas da Educação em Servi ço. Observou-se, também, que a Educação em Serviço dos HGI, incluiam, mais freqüentemente, as enfermeiras de outras unidades na realização dos seus programas.

Estes resultados demonstram, portanto, que o número de enfermeiras e a participação de outras enfermeiras interferem no desenvolvimento das diferentes fases e atividades da realizaçăo dos programas de preparo de pessoal.

No que diz respeito aos recursos materiais e área física para a execuçào dos programas observou-se que tanto os $\mathrm{HGl}$, como os HGII, dispunham de vários tipos de material didático, de sala de aula exclusiva para a Educação em Serviço, e utilizavam a unidade de internação como local para a realização dos programas.

CHIAVENATO' e NŨNEZ \& LUCKESI'" consideraram que o material didático favorece a aprendizagem, facilitando a compreensão do conteúdo apresentado e destacaram, entre eles, os audio-visuais e a biblioteca.

CAMARGOः e CASTRO ${ }^{\circ}$ referem que, quando a Educação em Serviço dispðe de salas de aulas equipadas adequadamente, hà maior facilidade para o desenvolvimento dos programas.

Vários autores demonstraram a importância da unidade de internação como local de treinamento, enfatizando que a unidade de trabalho é essencial para a aprendizagem, uma vez que permite ao funcionário vivenciar as atividades que irá desenvolver.

Evidenciou-se, também, que tanto os $4 \mathrm{HGl}(100 \%)$ como 7 dos $8 \mathrm{HGIl}$ $(87,50 \%)$ nāo contam com verba específica destinada exclusivamente para a Educaçào em Serviço.

Vários autores como CASTRO ${ }^{n}$, COOPER \& HORNBACK", DI VICENTI 14 e SILVA "x relalaranı ser importante, para a Educaçào em Serviço, a disponibilidade de verba própria, uma vez que isto facilita a aquisição de material didático. 
Quanto à caracterização da enfermeira responsável pela Educação em Serviço, observou-se que os HGI não diferiram, significativamente, dos HGIl, em relação a carga horária semanal, turno de trabalho e tempo de exercício na profissão.

Verificou-se que na maioria dos HGI e HGIl as enfermeiras trabalhavam de 30 até 40 horas semanais, no turno da manhã, exercendo a profissão há mais de 5 anos.

Por outro lado constatou-se que as enfermeiras dos HGI apresentaram maior tempo de atuação exclusivamente na Educação em Serviço ( 3 a 6 anos) que as dos HGII.

Estes resultados demonstram que o tempo de atuação da enfermeira responsável pela Educação em Serviço nessa área interfere na implementaçào das diferentes fases do programa de preparo de pessoal.

Em relação ao preparo da enfermeira responsável pela Educação em Serviço, verificou-se que tanto as dos $\mathrm{HGl}$ como as dos HGll fizeram algum outro tipo de curso após o curso de graduação, embora tenha observado que as dos HGI participaram de maior variedade de cursos.

Para COOPER \& HORNBACK", tem sido fundamental que a enfermeira responsável pela Educaçào em Serviço preocupe-se con seu auto-desenvolvimento; este autor considera tal requisito como essencial na seleçào de enfermeiras para a posição de coordenadora da Educação em Serviço.

Quanto às atividades desenvolvidas pela enfermeira responsável pela Educaçào em Serviço, verificou-se que tanto nos HGI como nos HGll as enfermeiras participaram do planejamento dos programas de preparo de pessoal. Por outro lado constatou-se que as enfermeiras dos HGll assumiam, lambém, a atividade supervisão de unidade, o mesmo não ocorrendo com os dos HGI.

Autores como NERY" e PERRODIN"s acreditam que a enfermeira que assume a responsabilidade da Educação em Serviço, paralelamente à supervisão de unidades de enfermagem, encontra maior dificuldade no desenvolvimento das atividades especificas da área de Educação.

\section{CONCLUSŌES}

Os resultados obtidos levaram às seguintes conclusðes:

- os hospitais pesquisados não executavam todas as fases e respectivas atividades dos programas de desenvolvimento de pessoal dè enfermagem apresentados pela autora;

- os hospitais que obtiveram maior número de pontos, ( $\mathrm{HGI}$ ), apresentaram, em relação aos de menor número de pontos, (HGII), maior contigente de enfermeiras na Educação em Serviço, desenvolvendo com maior frequência tanto as atividades que compðem as fases dos programas de preparo de pessoal, como as específicas da área de Educação em Serviço;

- o setor de Educação em Serviço, dos hospitais estudados dispunham de material didático e área física suficientes para realização dos programas. 
LEITE, M.M.J. Study on the programmes for preparation of nursing personnel. Implemented phases in clinical agencies of the Escola de Enfermagem da USP. Rev. Esc. Enf. USP, Såo Paulo, 22(2):189-198, Aug. 1988.

The author carries out a survey on the phases and on the activities which are part of the programmes for the development of nursing personnel, accomplished by the clinical agencies of the Escola de Enfer. magem da USP.

UNITERMS: Education, service. Nursing service hospital. Inservice training.

\section{REFERÊNCIAS BIBLIOGRÁFICAS}

1. AQUINO, C.P. de Administraçio de recursos bumanos: uma introdbcio. Såo Paulo, Atlas, 1979. p. 76-182.

2. BANDEIRA, N.Z. Desenvolvimento de recursos humanos: uma análise critica e uma proposição. Rev. Tecnol. Educ., Rio de Janeiro, 8(26):7-19, 1979.

3. BERGAMINI, C.W. Desenvolvimento de recursos humanos: uma estrattyla de desenvolvimento organizacional. Sao Paulo, Atlas, 1980. p. 37-41, 125-137.

4. CAMACHO, J. da S. Organização sistêmica de recursos humanos: uma proposta metodológica. Såo Paulo, 1981. p. 264-269. (Tese de doutorado - Pontificia Universidade Católica de Săo Paulo).

5. CAMARGO, C. de A. Como planejar programa de educaçăo em serviço para pessoal hospitalar. Rev. Paul. Hosp., Săo Paulo, 20(3):26-34, mar. 1972.

6. CASTRO, B.T. de. Consideraçðes gerais sobre educaçăo em serviço. Rev. Bras. Enf., Brasilia, 24 (5):182-207, jul./set. 1981.

7. CHIAVENATO, 1. Administraç̧o de recursos humanos. 2 ed. Săo Paulo, Atlas, 1980. v. 3, 278p.

8. . Administraçio: teoria, processo e prática. Săo Paulo, McGraw-Hill do Brasil. 1985, p. 3-34.

9. 343-350.

. Teoria geral da administração. São Paulo, McGraw-Hill do Brasil, 1979. v. 1, p.

10. COOMBS, P.H. Que é planejamento educacional? Cad. Pesq., Såo Paulo. (4):53-91, out. 1972.

11. COOPER, S.S. \& HORNBACK, M.S. Continuing nursing education. New York, McGraw-Hill, 1973. $251 \mathrm{p}$.

12. DAVIES, I.K. A organização do treinamento. Săo Paulo, McGraw-Hill do Brasil, 1976. 145 p.

13. DEIMAN, P.A. Programa de educacion en serviço. In: ORGANIZAÇẢo PANAMERICANA DE LA SALUD. Administracion de serviços de enfermeria. Washington, 1971. p. 1-12. (Informes de enfermeria, $n$. 9).

14. DI VICENTI, M. Administering nursing service. Boston, Little Brown, 1972. p. 153-169.

15. DRAKE, R. \& SMITH, P. Ciéncia do comportamento å indústria. Sáo Paulo, McGraw-Hill do Brasil, 1976. p. 33-56.

16. ESCOBAR, F. Principios de aprendizaje del adulto en programas de education continua. Educ. Med. Salud, Washington, 12(2):135-148, abr./jul. 1978.

17. FONTES, L.B. Manual do treinamento na empresa moderna. Sào Paulo, Atlas, 1971. p. 39-84.

18. HEADRICKS, M.M. Sense or norisense? J. Contin. Educ. Nurs., Thorofare, 14(5):13-15, sept./ oct. 1983.

19. HORN, R.S. Planning inservice education in small hospitals. Superv. Nurse, Chicago, 12(1):3841, Jan. 1981.

20. KATZ, F.M. La producción de recursos humanos y los planificadores de la salud, In: HALL, T. L. \& MEJIA, A. Planificacion del personal de salud. Genebra, Organizacion Mundial de la Salud, 1979. cap. 6, p. 151-166.

21. KURCGANT, P. Educaçð̃o contínua: necessidades sentidas e evidenciadas por enfermeiros e obstetrizes do Municipio de Såo Paulo, 1977. Såo Paulo, 1978. p. 6-10. (Dissertaçăo de mestrado - Escola de Enfermagem da Universidade de Săo Paulo).

22. LEWY, A. Avaliaçào de currículo. Såo Paulo, EPU, 1979. p. 78-79.

23. LITTERER, J.A. And́lise das organizações. São Paulo, Atlas, 1970. p. 458-460.

24. LUCKESI, C.C. Avaliaçào educacional: pressupostos conceituais. Rev. Teenol. Educ., Rio de Janeiro, 7(24):5-8, 1979.

25. MARCONDES, R.S. Treinamento de pessoal em hospitais. Rev. Paul. Hosp., Sao Paulo, 26(10): 436-440, out. 1978. 
26. MARRINER, A. Continuing education in nursing. Superv. Nurse, Chicago, 6(6):20-22, june 1975.

27. McGREGOR, D. Motivação e liderança. São Paulo, Brasilense, 1973. p. 5-15.

28. MEJIA, A. \& FÜLOP, T. Planificación del personal de salud: examen general. In: HALL, T.L. \& MEJÍA, A. Planificación del personal de salud. Ginebra, Organizacion Mundial de la Salud, 1979. cap. 1, p. 9-34.

29. MOTTA, F.C.P. Teoria geral da administração: uma introduç̄ono. 3. ed. Sảo Paulo, Pioneira, 1975. $146 \mathrm{p}$.

30. NASCIMENTO, K.T. A evolução conceptual de administração: implicaçðes para a formulação dos papês e funçð̌es essenciais de um executivo. Rev. Adm. Publ., Såo Paulo, 6(2):5-52, abr./jun. 1972.

31. NERY, I.S. \& FERNANDES, W. Supervisão em enfermagem. Enf. Atual, Rio de Janeiro, 1(2): 18-24, mar./abr. 1978.

32. NUNEZ, R.S. \& LUCKESI, M.A.V. Educação em serviço fatos de desenvolvimento de recursos humanos em enfermagem. Rev. Bras. Enf., Brasilia, 33(1):54-80, jan./mar, 1980.

33. PARO, V.H. Conceito, justificativa e fases do planejamento. Cad. Pesq. Såo Paulo, (8):63-76, set. 1976.

34. PERRODIN, C.M. Supervision de los serviços de enfermeria. México, Interamericana, 1965. 107 p.

35. PETERS, T. \& WATERMAN JÚNIOR, R.H. Vencendo a crise. 3.ed. Sảo Paulo, Arxpec \& Row do Brasil, 1983. p. 101-110.

36. POPIEL, E.S. Nursing and the process of continuingeducation. 2. ed. Saint Louis, Mosby, 1977. p. 47-73.

37. SANTOS, O. de B. Orientaçào e desenvolvimento humano. Såo Paulo, Pioneira, 1978. p. 9-10, 139-145.

38. SILVA, A.L.C. Caracterizaçăo das formas de progresso para competência continuada em enfermagem. Rev. Bras. Enf., Brasilia, 33(4):453-461, out./dez. 1980.

39. SMITH, H.C. Psicologia do comportamento na indústria. Såo Paulo, Atlas, 1976. p. 107-118.

40. TEJADA-DE-RIVERO, D. Introducción. In: HALl, T.L. \& MEJİA, A. Planificación del personal de salud. Ginebra, Organizacion Mundial de la Salud, 1979. p. 5-8.

41. TOLEDO, P. Manual de administraçio de pessoal. 3. ed. Sào Paulo, Atlas, 1969. p. 20-37.

42. TURRA, C.M.V. et alii. Planejamento de ensino e avaliação. 10. ed. Porto Alegre, Pontifícia Universidade Católica, 1975. p. 25-120.

43. WOLANIN, M.O. Factors leading to effectiveness of continuing education programs. J. Contin. Educ. Nars., Thorofare, 4(6):14-9, Nov./Dec. 1973. 